\title{
A class of internally irreversible refrigeration cycles
}

\author{
Mohand A Ait-Ali \\ Département Génie Mécanique, Ecole Nationale Polytechnique, BP 182 , \\ Hassen-Badi, Alger 16100, Algeria and University of Michigan MEAM Department, \\ 2212 G G Brown Building, Ann Arbor, MI 48109-2125, USA
}

Received 20 June 1995, in final form 27 September 1995

\begin{abstract}
A Carnot-like irreversible refrigeration cycle is modelled with two isothermal and two non-adiabatic, irreversible processes. The generic source of internal irreversibility, measured by the Clausius inequality, is a general irreversibility term which could include any heat leaks into the Joule-Thompson expansion valve, the evaporator and compressor cold boxes. This cycle is optimized first for maximum refrigeration power and maximum refrigeration load, then for maximum coefficient of performance. Its performances are compared with those of the endoreversible refrigeration cycle, based on a propane stage of a classical cascade liquefaction cycle example. Both cycle models achieve optimum power and maximum refrigeration load at nearly the same refrigeration temperature, but only the coefficient of performance of the irreversible refrigeration cycle reaches a maximum. Moreover, its prediction of heat conductance allocation between evaporator and condenser appears to be not only more conservative, but also more realistic for actual design considerations of refrigeration cycles.
\end{abstract}

\section{Nomenclature}

A Heat transfer area

$A_{m c} \quad$ Arithmetic mean temperature difference in the condenser

$A_{m e} \quad$ Arithmetic mean temperature difference in the evaporator

$C_{e} \quad$ Heat conductance in the evaporator

$C_{h} \quad$ Heat conductance in the condenser

$C_{t} \quad$ Overall cycle heat conductance

COP Coefficient of performance

$F \quad$ Irreversibility coefficient

$Q_{c} \quad$ Evaporator heat rate

$Q_{h} \quad$ Condenser heat rate

$Q_{l c} \quad$ Heat leak into the compressor

$Q_{l v} \quad$ Heat leak into the expansion valve

$P \quad$ Refrigeration power

$s \quad$ Entropy

$T_{c} \quad$ Refrigerant arithmetic mean temperature in the condenser

$T_{e} \quad$ Refrigerant arithmetic mean temperature in the evaporator

$T_{c e} \quad$ Refrigeration temperature

$T_{h c} \quad$ Refrigerant higher isotherm

$U \quad$ Overall heat transfer coefficient

$\Delta \quad$ Refrigerant operating temperature range

$\sigma \quad$ Cycle internal rate of entropy production

\author{
Subscripts \\ $c$ for Carnot \\ $e$ for endoreversible \\ $i$ for irreversible.
}

\section{Introduction}

The endoreversible refrigeration cycle model, with a specified overall heat transfer conductance and a refrigerant temperature range, has been shown [1] to predict very close maxima for refrigeration load and refrigeration power, as well as for heat rejection load (in the heat pump case). However, its coefficient of performance $\left(\mathrm{COP}_{e}\right)$ is predicted to have relatively high values and a simply monotonic increase with respect to the refrigeration temperature. From basic thermodynamics considerations, one would expect the coefficient of performance of irreversible refrigeration cycles $\left(\mathrm{COP}_{i}\right)$ to decrease towards zero for very low refrigeration temperatures, but also when refrigeration temperatures approach the heat sink temperature since the compression power has to be at least large enough to overcome internal irreversibilities. Another investigation [2] of endoreversible refrigeration cycles with several heat transfer laws showed the existence of an optimum coefficient of performance with the rate of refrigeration. However simple or complex, endoreversible cycle models do not incorporate enough of the essential physics of actual cycles in order to produce realistic predictions.

Furthermore, it is not known how the predicted average mean temperature differences in the evaporator and condenser heat exchangers, and therefore heat transfer areas, are affected by internal irreversibilities. In a recent [3] evaluation of a Carnot-like irreversible power cycle model, optimized for maximum power and maximum thermal efficiency, it was found that the model predictions 
of these maxima are very sensitive to the heat transfer conductance allocation to the boiler and condenser; the relative size of these heat exchangers actually determines the optimality either of maximum power or of maximum efficiency.

Consequently, and for all practical purposes, the predicted heat rates and heat transfer conductances, and therefore heat transfer areas as well as refrigeration power figures, are expected to be significantly different for irreversible refrigeration cycles. It thus appears that a more realistic, and yet still simple, irreversible refrigeration model cycle is needed to predict the coefficient of performance with refrigeration temperature better, as well as to provide a more accurate heat transfer conductance allocation for the evaporator and condenser. Improved predictions of performance and equipment size are needed in order to obtain more reliable estimates of investment and operating costs before a detailed project evaluation.

In this paper, it is proposed to investigate the influence of internal irreversibilities in general on a Carnot-like refrigeration cycle performance, in terms of maximum power and refrigeration load, as well as maximum coefficient of performance. These irreversibilities could be produced by mechanical or fluid friction, fluid expansion in the Joule-Thompson valve and other orifices, and heat leaks into the evaporator and compressor cold boxes.

The problem objectives consist of maximizing with respect to the refrigeration temperature first the compression power and refrigeration load and then the coefficient of performance, subject to a specified overall heat conductance and an overall internal rate of entropy production. Since the problem has been found to be unbounded [1,4] when considering the two refrigerant isotherms as degrees of freedom, a refrigerant boiling temperature range is specified as a necessary bounding constraint. As was argued earlier for the case of the endoreversible refrigeration cycle, this temperature range is defined as the difference between the refrigerant mean condensing temperature and its mean evaporation temperature, as would be considered when selecting any candidate refrigerant.

The overall heat conductance equality constraint imposes a finite size for the condenser and evaporator, and therefore defines a certain heat exchanger investment to be made for which the optimum values of the objective functions are to be found. A Clausius equality constraint specifies the overall internal entropy rate of production for which the objectives are considered. This entropy rate of production is taken to be proportional to the overall heat conductance, as well as to the ratio of the refrigerant temperature range and the upper isotherm. Since the heat transfer conductance and entropy rate of production are both expressed in power units per kelvin, their ratio is conveniently dimensionless.

The remainder of this paper is organized as follows. After a qualitative discussion of the cycle $T-S$ diagram, the two equality constraint equations are solved as a linear system for the heat rates in the evaporator and condenser. Then, the maximum power and maximum coefficient of performance objectives are formulated as unconstrained optimization problems with respect to the refrigeration

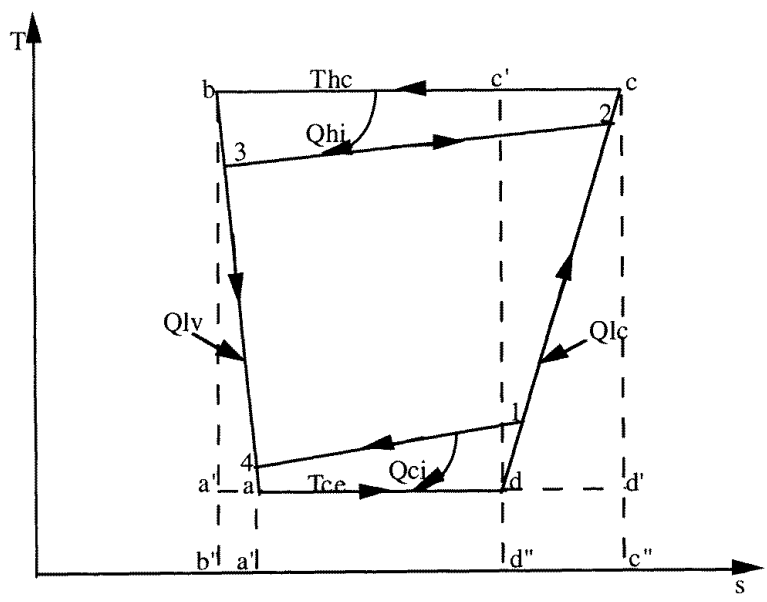

Figure 1. The irreversible refrigeration cycle $T-S$ diagram.

temperature as the unique free variable. Because the optimum refrigeration temperature is obtained as an implicit relation of the design parameters, the optimum values of the objective functions are described and analysed by means of a numerical example related to a classical cascade cycle natural gas liquefaction cycle as simulated in [5].

\section{Formulation of the problem}

In refrigeration cycle analysis, it is convenient to assume that the compressor suction operates at a fixed, near saturation vapour state and that the Joule-Thompson valve inlet is in a nearly liquid saturated state. Fluid friction and uncompensated fluid expansion can be regarded as irreversibilities which add up to heat leaks into the JouleThompson valve and evaporator cold box; their effect is to decrease the useful refrigeration load. Internal irreversibilities in the compressor and heat leaks into its suction line increase the compressor power and, consequently, the condenser heat load. Clearly, heat leaks and power dissipation irreversibilities can be lumped together and measured with the same yard stick for the purpose of analysing internal irreversibility effects on refrigeration cycle performance. The problem is now formulated from basic thermodynamics considerations of irreversibilities and heat leaks as accounted for in the Clausius inequality.

The problem formulation is based on the cycle $T-S$ diagram of figure 1, in which the heat source supplies the time-averaged heat rate $Q_{c i}$ at a temperature varying from $T_{1}$ to $T_{4}$, for which the arithmetic mean value is $T_{e}$, in a counterflow boiler heat exchanger and the heat sink receives the time-averaged heat rate $Q_{h i}$, while its temperature increases from $T_{3}$ to $T_{2}$, for which the arithmetic mean value is $T_{c}$, in a counterflow condenser heat exchanger.

This refrigeration cycle is composed of the following four processes.

(i) From d to c: a non-adiabatic irreversible compression process, with $Q_{l c}$ as the time-averaged heat 
leak into the compressor, for which the net entropy production term is equal to $s_{c}-s_{d}$.

(ii) From $\mathrm{c}$ to $\mathrm{b}$ : an isothermal heat transfer process for which the entropy decreases from $s_{c}$ to $s_{b}$ as a result of heat transfer from the condensing fluid into the heat sink.

(iii) From $\mathrm{b}$ to $\mathrm{a}$ : a non-adiabatic irreversible expansion process, with $Q_{l v}$ as the time-averaged heat leak into the expansion valve and evaporator, for which the net entropy production term is equal to $s_{a}-s_{b}$.

(iv) From a to d: an isothermal process for which the entropy increases from $s_{a}$ to $s_{d}$ as a result of heat transfer into the evaporating fluid.

Note that, in actual refrigeration cycles, condensation of the refrigerant occurs after some de-superheating, whereas evaporation is followed by some superheating prior to compression; the real heat transfer processes are therefore neither isotherms nor isobars; the isotherms considered here are averaged values to be obtained as ratios of heat load to entropy change for each heat transfer process.

If one were to compare this irreversible cycle with the endoreversible cycle represented by processes $a^{\prime}-d^{\prime}-c-b-$ $\mathrm{a}^{\prime}$, one would notice that the heat rate $Q_{h i}$, rejected to the heat sink, is proportional to the rectangular area $b-b^{\prime \prime}-c^{\prime \prime}-$ c, and common to both cycles. However, the heat rate $Q_{c i}$, which is the useful refrigeration load received from the heat source, is always less for the irreversible cycle. Consequently, although the refrigeration power supplied to the irreversible cycle is less than that of the endoreversible cycle for the same $Q_{h i}$, the useful refrigeration load will be even less and the coefficient of performance will be smaller than that for the corresponding endoreversible cycle. This means that the endoreversible refrigeration cycle model overestimates coefficients of performance of actual refrigeration cycles.

The irreversible refrigeration cycle to be investigated is constrained to have finite heat transfer rates $Q_{h i}$ and $Q_{c i}$, subject to the following specification of the overall heat conductance $C_{t}$

$$
\frac{Q_{h i}}{T_{h c}-T_{c}}+\frac{Q_{c i}}{T_{e}-T_{c e}}=C_{t}
$$

where $T_{c}$ and $T_{e}$ are the respective arithmetic mean temperatures of the heat sink and the heat source, defined by

$$
\begin{aligned}
& T_{c}=\frac{T_{2}+T_{3}}{2} \\
& T_{e}=\frac{T_{1}+T_{4}}{2} .
\end{aligned}
$$

The irreversible cycle must also satisfy the Clausius inequality, written here as an equality, where $T_{c e}$ is the refrigeration temperature and $T_{h c}$ the refrigerant condensing temperature

$$
\frac{-Q_{h i}}{T_{h c}}+\frac{Q_{c i}}{T_{c e}}=-\sigma
$$

where the internal entropy production term $\sigma$ is now defined by the following equality, using geometric considerations from the $T-S$ diagram of figure 1 :

$$
\sigma=\left(s_{c}-s_{d}\right)+\left(s_{a}-s_{b}\right) .
$$

The heat rates $Q_{h i}$ and $Q_{c i}$ used here are integrated mean values obtained over the cycle period of time in order to be consistent with the Carnot cycle reversibility definition. Equations (1) and (4) are solved as a linear system for $Q_{h i} / C_{t}$ and $Q_{c i} / C_{t}$; they yield

$$
\begin{aligned}
\frac{Q_{h i}}{C_{t}} & =\frac{1}{D}\left(\frac{1}{T_{c e}}+\frac{\sigma}{C_{t} A_{m e}}\right) \\
\frac{Q_{c i}}{C_{t}} & =\frac{1}{D}\left(\frac{1}{T_{h c}}-\frac{\sigma}{C_{t} A_{m c}}\right)
\end{aligned}
$$

where the common denominator $D$ is the linear system determinant expressed by

$$
D=\frac{1}{T_{c e} A_{m c}}+\frac{1}{T_{h c} A_{m e}}
$$

and the arithmetic mean temperature differences in the boiler and condenser, respectively, are expressed by

$$
\begin{aligned}
& A_{m c}=T_{h c}-T_{c} \\
& A_{m e}=T_{e}-T_{c e} .
\end{aligned}
$$

Note that use of arithmetic mean temperature differences, instead of logarithmic mean temperature differences (LMTDs), in actual heat transfer calculations will somewhat underestimate the heat transfer area, depending on how much the temperature profiles diverge; they are used here as a convenience to simplify the algebra and yield analytical closed form solutions.

The irreversible cycle refrigeration power $P_{i}$ and coefficient of performance $\mathrm{COP}_{i}$ are now expressed using the first law:

$$
\begin{gathered}
\frac{P_{i}}{C_{t}}=\frac{1}{D}\left[\frac{1}{T_{c e}}+\frac{1}{T_{h c}}+F \frac{\Delta}{T_{h c}}\left(\frac{1}{A_{m c}}+\frac{1}{A_{m e}}\right)\right] \\
\mathrm{COP}_{i}=\frac{A_{m c}-F \Delta}{\Delta A_{m c} / T_{c e}+F \Delta\left(1+A_{m c} / A_{m e}\right)} .
\end{gathered}
$$

The following model for the internal production rate of entropy $\sigma$ is adopted:

$$
\sigma / C_{t}=F\left(\Delta / T_{h c}\right)
$$

where $F$ is a dimensionless numerical coefficient. In the numerical example to be considered in section 7 , an arbitrary value of $F=0.02$ will be retained in order to compare the refrigeration power loss to be estimated here with the power loss obtained in a previous study [3] on internally irreversible power cycles; but, ultimately, some relationship between isentropic coefficients of the turbine and compressors and internal rates of entropy production $\sigma$ could be developed to fit observed data from actual refrigeration cycles whenever available.

The refrigerant operating temperature range $\Delta$ is defined by

$$
\Delta=T_{h c}-T_{c e} .
$$

This temperature range is equal to the difference between the mean condensing temperature retained for the refrigerant considered and its evaporation temperature, as would be done when selecting any candidate refrigerant for a particular application. 


\section{The maximum refrigeration power objective}

Although a minimum refrigeration power objective would be more appropriate for refrigeration cycles, a maximum power objective will be sought instead, because it is already known [1] that the refrigeration power of an endoreversible cycle achieves a maximum, and not a minimum; moreover, this maximum is reached at nearly the same refrigeration temperature as that at which a maximum refrigeration load is also achieved.

The unconstrained maximization of this objective as defined by equations (11) and (8), with respect to $T_{c e}$, requires that its first derivative with respect to this free variable be zero. The resulting necessary (and sufficient) condition is

$$
\begin{aligned}
& A_{m c}^{2}\left(T_{e}-F T_{h c}\right)+A_{m e}^{2}\left(-T_{c}+F T_{c e}\right) \\
= & F \Delta A_{m c} A_{m e}\left[1+T_{c e}\left(\frac{1}{A_{m c}}+\frac{1}{A_{m e}}\right)\right] .
\end{aligned}
$$

When $F=0$, this optimality condition reduces to that of the endoreversible refrigeration cycle, that is

$$
\left(A_{m c} / A_{m e}\right)^{2}=T_{c} / T_{e} .
$$

When the irreversibility coefficient is different from zero, equation (15) can only be solved numerically; but it can be seen that this ratio of arithmetic mean temperature differences, or equivalently the ratio of heat transfer conductances, will be larger for irreversible cycles. This will be shown in the numerical example to be considered in section 7 .

\section{The maximum refrigeration load objective}

The unconstrained maximization of this objective as defined by equations (7) and (8), with respect to $T_{c e}$, requires that its first derivative with respect to this free variable be zero. The resulting necessary (and sufficient) condition for a maximum is

$\frac{A_{m c}^{2}}{A_{m e}^{2}}=\frac{T_{h c}^{1 / 2}}{T_{c e}^{1 / 2}}\left(\frac{A_{m c}}{T_{c e}}+\frac{T_{c}}{T_{h c}}\right)+F \Delta\left[\frac{\Delta}{T_{c e}^{2}}-\left(\frac{A_{m e}+A_{m e}}{A_{m e}^{2}}\right)\right]$.

When $F=0$, this optimality condition reduces to that of the endoreversible refrigeration cycle, that is

$$
\frac{A_{m c}^{2}}{A_{m e}^{2}}=\frac{T_{h c}^{1 / 2}}{T_{c e}^{1 / 2}}\left(\frac{A_{m c}}{T_{c e}}+\frac{T_{c}}{T_{h c}}\right) .
$$

When the irreversibility coefficient $F$ is different from zero, equation (17) can only be solved numerically; but it can be seen that this ratio of mean temperature differences will be larger for irreversible cycles than for endoreversible cycles; this will be shown in the numerical example.

\section{The maximum coefficient of performance objective}

The unconstrained maximization of this objective as defined by equation (12), with respect to $T_{c e}$, requires that its first derivative with respect to this free variable be zero.
The resulting necessary (and sufficient) condition for a maximum is

$$
\frac{A_{m c}^{2}}{T_{c e}^{2}}=F\left(1+\frac{\Delta}{T_{c e}}\right)\left(\frac{A_{m c}}{A_{m e}}-1\right)-F^{2} \frac{\Delta}{A_{m e}}\left(1+\frac{A_{m c}}{A_{m e}}\right) .
$$

For an endoreversible cycle, $F=0$ and this optimality condition reduces to $A_{m c}=0$, that is, $T_{h c}=T_{c}$; this solution corresponds to the Carnot cycle coefficient of performance, since the endoreversible cycle $\mathrm{COP}_{e}$ has no maximum.

Like the optimality expressions derived above, this optimality relation cannot be solved analytically; but it can be observed that feasibility requires that the arithmetic mean temperature difference in the condenser should be at least as large as that in the evaporator according to this model; furthermore, since the first right-hand side term of equation (19) should be larger than the second, feasible values of the mean temperature differences should be such that

$$
F<\frac{A_{m e}}{\Delta} \frac{T_{h c}}{T_{c e}} \frac{A_{m c}-A_{m e}}{A_{m c}+A_{m e}} .
$$

This relation requires some knowledge of the feasible upper bound of the irreversibility factor $F$.

\section{Feasible limits of the irreversibility coefficient $\boldsymbol{F}$}

The lower bound of the irreversibility factor $F$ is zero, which corresponds to an endoreversible cycle. At the feasible upper bound of the irreversibility factor $F$, the refrigeration heat rate $Q_{c i}$ becomes zero, as can be observed from figure 1. Using this condition in equation (7) gives the following double inequality:

$$
0 \leq F \leq \frac{A_{m c}}{\Delta}=\frac{A_{m c}}{T_{c e}} \mathrm{COP}_{e}
$$

As expressed, the feasible lower and upper bounds of the irreversibility factor $F$ are respectively zero and some fraction of the endoreversible coefficient of performance for which an irreversible cycle version is considered.

Alternatively, the feasible upper bound $F_{\max }$ can be expressed in terms of the Carnot $\mathrm{COP}_{c}$ and the endoreversible cycle $\mathrm{COP}_{e}$ as

$$
F_{\text {max }}=\left(1+\mathrm{COP}_{e}\right)-\frac{\delta}{\Delta}\left(1+\mathrm{COP}_{c}\right)
$$

where $\delta$ is the temperature potential difference $T_{c}-T_{e}$ given in any problem and $\Delta$ is a specified optimization parameter of the problem. $F_{\max }$ can thus be specified by this linear combination of the coefficients of performance of the heat pump case. It is seen that the irreversibility factor $F$ is identically equal to zero for the Carnot refrigeration cycle, because the ratio $\delta / \Delta$ is equal to unity and $\mathrm{COP}_{e}=\mathrm{COP}_{c}$. To summarize this point, the value of the irreversibility factor $F$ that can be considered in a cycle design must lie between zero and $F_{\max }$, as given by equation (21).

However, knowledge of the limits of the factor $F$ does not determine how the value of $F$ varies between them. By 
drawing a parallel with the isentropic efficiency coefficient used in power cycles, we could relate the value of $F$ for which a given refrigeration cycle is to be optimized to its maximum feasible value. From equation $(22), F_{\max }$ is seen to be a function of the parameter $\Delta$ to be specified and the free variable $T_{c e}$ :

$$
F_{\text {max }}=1+\frac{T_{c e}}{\Delta}-\frac{\delta}{\Delta}\left(1+\mathrm{COP}_{c}\right)=1-\frac{T_{c}-T_{c e}}{\Delta} .
$$

This function increases monotonically with $\Delta$ and $T_{c e}$. Its maximum is attained with the largest feasible value of $T_{c e}<T_{e}$. Its upper bound is given by

$$
F_{\text {max }} \leq 1-\frac{\delta}{\Delta}
$$

The ratio $F / F_{\max }$ is now defined by $\rho$ and expressed as

$$
\rho=\frac{F}{F_{\max }}=\frac{\Delta F}{\Delta-\delta}
$$

or equivalently, if the value of $\rho$ for which to optimize is fixed, the corresponding value of the irreversibility coefficient $F$ is expressed by

$$
F=\rho\left(1-\frac{\delta}{\Delta}\right) .
$$

For instance, in the example to follow, a value of $F=0.02$ corresponds to about $\rho=0.05$.

The goal of this irreversible refrigeration cycle model is to predict optimum power requirements of actual refrigeration cycles better for a given heat transfer conductance. If a parallel is again drawn with power cycles, in which the turbine inlet and condensation temperatures are usually specified parameters, then the turbine power is calculated simply by using its isentropic coefficient given by the constructor.

The ratio of isentropic to actual power requirements is equal to the ratio of the irreversible cycle $\mathrm{COP}_{e}$ and the Carnot cycle $\mathrm{COP}_{c}$. This ratio is expressed here as the product of a temperature effectiveness and a compressor isentropic efficiency:

$$
\frac{\mathrm{COP}_{i}}{\mathrm{COP}_{c}}=\eta_{c} \frac{T_{h c}}{T_{c}} \frac{\delta}{\Delta}=\eta_{c} \frac{1-T_{e} / T_{c}}{1-T_{c e} / T_{h c}} .
$$

Alternatively, this ratio is a function of the condensation temperature $T_{c e}$ when considering equation (14). In the following example, it can be seen that the temperature effectiveness varies from 0.775 to 0.800 , whereas the corresponding compressor efficiency varies from 61 to $63 \%$.

\section{A numerical example}

This numerical example will illustrate the variations of the objective functions considered, with respect to the free variable $T_{c e}$, for the propane low-pressure refrigeration stage of a classical cascade liquefaction cycle described by Kao et al [5]. In this refrigeration stage, natural gas is cooled from 260 to $241 \mathrm{~K}$; the problem parameters are

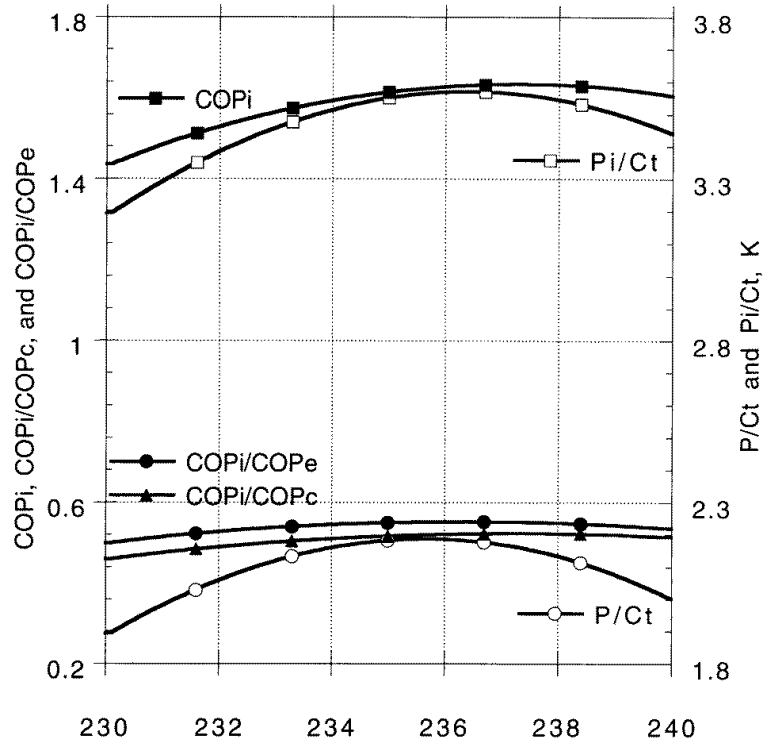

Figure 2. Refrigeration power and coefficient of performance versus refrigeration temperature.

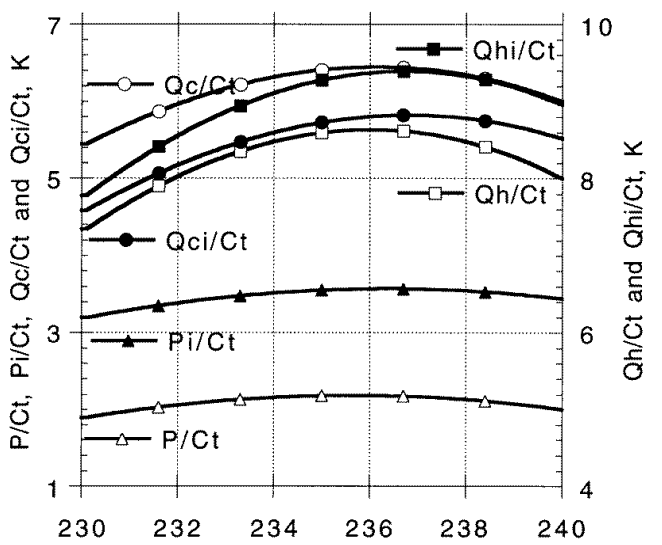

Figure 3. Power and heat rates versus refrigeration temperature.

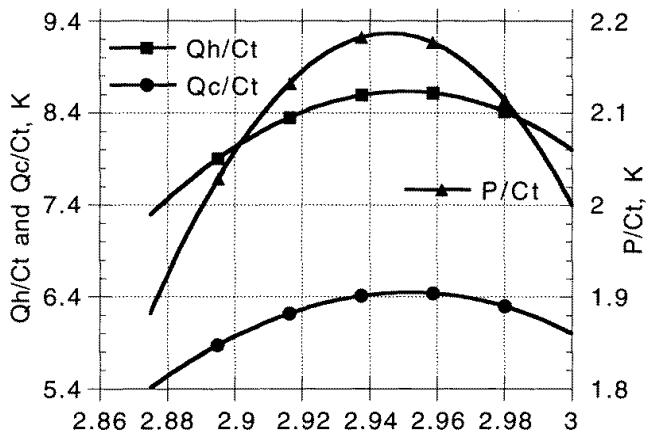

Figure 4. Endoreversible refrigeration cycle power and heat rates versus $\mathrm{COP}_{e}$.

taken as $T_{e}=250 \mathrm{~K}, T_{c}=300 \mathrm{~K}$, and $\Delta=80 \mathrm{~K}$. The results of these calculations are shown in figures 2-6; the internal irreversibilities are calculated with $F=0.02$.

Figure 2 shows the variations of the refrigeration 


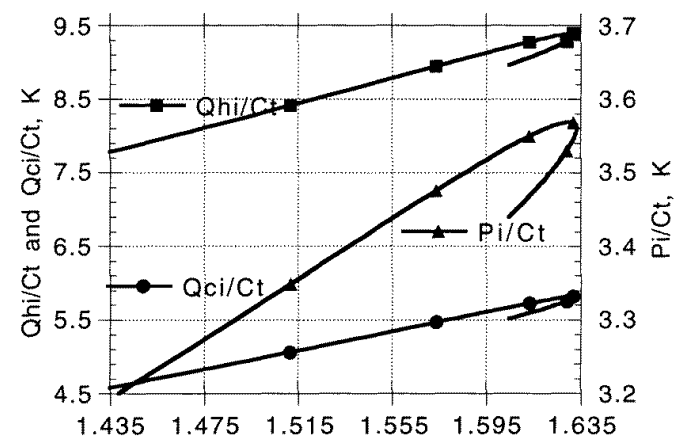

Figure 5. Irreversible refrigeration cycle power and heat rates versus $\mathrm{COP}_{i}$.

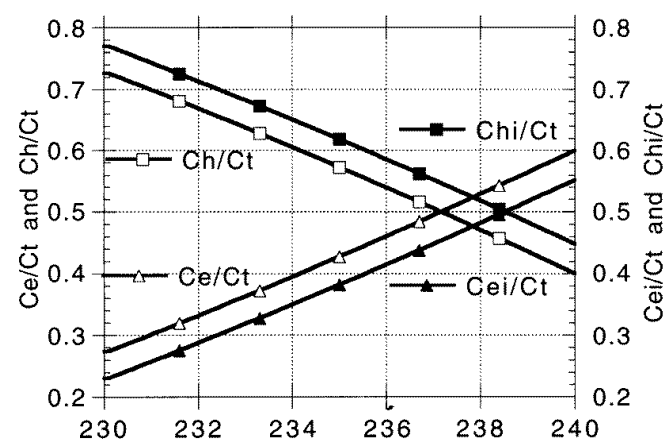

Figure 6. Heat transfer conductance versus refrigeration temperature.

power and coefficient of performance with respect to the refrigeration temperature $T_{c e}$. The maximum of the refrigeration power occurs at $236.4 \mathrm{~K}$ for the irreversible cycle model and at $235.6 \mathrm{~K}$ for the endoreversible model; the respective maxima per unit overall heat conductance are 3.569 and $2.186 \mathrm{~K}$, in $\mathrm{kW} /\left(\mathrm{kW} \mathrm{K}^{-1}\right)$. The entropy rate of production per unit overall heat conductance $\sigma / C_{t}$ at this maximum is only 0.00506 ; but it increases the refrigeration power by as much as $63.3 \%$ relative to the endoreversible cycle refrigeration power.

The coefficient of performance $\mathrm{COP}_{i}$ of the irreversible cycle reaches its maximum value, 1.6335 , at $237.4 \mathrm{~K}$; the corresponding value of $\mathrm{COP}_{e}$, for the endoreversible cycle is 2.9675. At this temperature of $-35.8{ }^{\circ} \mathrm{C}$, the ratio of $\mathrm{COP}_{i}$ to the Carnot cycle coefficient of performance $\mathrm{COP}_{c}$ is only 0.523 . This is a useful parameter to consider when comparing actual versus Carnot refrigeration cycles.

Figure 3 compares refrigeration power and heat rates for the irreversible and endoreversible cycles. The maxima of power and heat rates occur at near the same temperature for each cycle, although at some $0.8 \mathrm{~K}$ higher for the irreversible cycle. The maximum refrigeration load occurs at $236.8 \mathrm{~K}$ for the irreversible cycle and at 236.2 for the endoreversible cycle. Internal irreversibility thus increases the optimum refrigeration temperature only slightly both for refrigeration power and for refrigeration load.

Figure 4 shows the variations in refrigeration power, refrigeration load and rejection heat load for the endoreversible cycle with respect to the coefficient of performance; power is seen to vary more sharply than do refrigeration load and rejection heat load. The purpose of figure 4 is to contrast with the behaviour displayed in figure 5 by the variation of power and heat rates of the irreversible cycle; these are shaped like 'canes' and double-valued, near the maximum value of the coefficient of performance.

Figure 6 compares the distribution of the overall heat conductance $C_{t}$ between the evaporator and condenser conductances, both for the endoreversible and for the irreversible cycles. It clearly shows that internal irreversibilities increase the condenser size relative to the evaporator size. At maximum refrigeration load, their ratio is 0.861 .

As would be expected, it was found in this investigation that the choice of the refrigerant operating temperature range $\Delta$ strongly influences the optimum solution of the problem. The larger the value of $\Delta$, the larger the refrigeration power per unit overall heat conductance. For $\Delta=100 \mathrm{~K}$, for instance, only the coefficient of performance $\mathrm{COP}_{i}$ achieves a maximum at a refrigeration temperature of $230.1 \mathrm{~K}$; the optimum temperature for maximum refrigeration load will be below $230 \mathrm{~K}$, which would not be feasible because it would correspond to a normal boiling pressure below 1 bar for propane. At $230 \mathrm{~K}$, for this case, the refrigeration power is increased by $72.3 \%$. Hence, designing for a large temperature range $\Delta$ means that the optimum solution will be biased towards lower refrigeration temperatures and larger refrigeration power per unit overall heat conductance. Consequently, several feasible alternatives with different values of $\Delta$ will have to be compared for power-related cost and heat exchanger cost before recommending a particular solution for any given project.

\section{Conclusion}

A Carnot-like, internally irreversible refrigeration cycle has been modelled using the Clausius entropy inequality. It has been optimized with respect to the refrigeration temperature, first for maximum power and maximum refrigeration load, then for maximum coefficient of performance. The results obtained for a specified refrigerant temperature range show that this irreversible cycle has flat and very close maxima for power and refrigeration load, just as the endoreversible cycle does, but at a slightly higher refrigeration temperature. It also achieves a maximum value for its coefficient of performance, which the endoreversible cycle does not. This is the distinguishing feature of this internally irreversible refrigeration cycle.

The particular value specified for the refrigerant temperature range, as a necessary bounding constraint, actually determines whether the objective functions considered achieve a feasible maximum. Moreover, a large value of this parameter specifies a correspondingly large temperature difference for the sum of the condenser and evaporator mean temperature differences. Consequently, the resulting optimum solution is biased not only towards a larger refrigeration power, but also towards a larger 
refrigeration load per unit overall heat conductance. The maximum potential temperature difference to overcome for a single-stage refrigeration cycle being typically $60 \mathrm{~K}$ and the maximum operating temperature range for a refrigerant being typically $80 \mathrm{~K}$, these two parameters strongly influence the actual cycle coefficient of performance.

\section{Acknowledgments}

The author gratefully acknowledges the support of the
MEAM Department of the University of Michigan, Ann Arbor, for the duration of this research.

\section{References}

[1] Ait-Ali M 1995 J. Appl. Phys. 773231

[2] Zijun Yan and Jincan Chen 1990 J. Phys. D: Appl. Phys. 23 136

[3] Ait-Ali M 1995 J. Appl. Phys. 784313

[4] Blanchard C H 1982 J. Appl. Phys. 512471

[5] Kao R L, Waterman W W and Bukacek R F 1976 ASME Winter Annual Meeting, New York, December 5 